hep-th/0606218

DAMTP-2006-44

\title{
Two-charge small black hole entropy: String-loops and multi-strings
}

\author{
Aninda Sinha ${ }^{1}$ and Nemani V. Suryanarayana ${ }^{2}$ \\ 1 Department of Applied Mathematics and Theoretical Physics, \\ Wilberforce Road, Cambridge CB3 OWA, U.K. \\ E-mail: A.Sinha@damtp.cam.ac.uk \\ ${ }^{2}$ Perimeter Institute for Theoretical Physics, \\ 31 Caroline Street North, Waterloo, ON, N2L 2Y5, Canada \\ E-mail: vnemani@perimeterinstitute.ca
}

July 18, 2018

\begin{abstract}
We investigate the inclusion of 10-dimensional string loop corrections to the entropy function of twocharge extremal small black holes of the heterotic string theory compactified on $S^{1} \times T^{5}$ and show that the entropy is given by $\pi \sqrt{a q_{1} q_{2}+b q_{1}}$ where $q_{1}$ and $q_{2}$ are the charges with $q_{1} \gg q_{2} \gg 1$ and $a$ and $b$ are constants. Incorporating certain multi-string states into the microstate counting, we show that the new statistical entropy is consistent with the macroscopic scaling for one and two units of momentum (winding) and large winding (momentum). We discuss our scaling from the point of view of related $A d S_{3}$ central charge and counting of chiral primaries in superconformal quantum mechanics as well.
\end{abstract}




\section{Contents}

1 Introduction 2

\begin{tabular}{|l}
2 A scaling argument \\
\hline
\end{tabular}

$\begin{array}{lll}3 & \text { Microstate re-counting } & 7\end{array}$

3.1 Multi-string Partition function . . . . . . . . . . . . . . . . 8

3.2 Example 1: $n=1 . w \gg 1 \ldots \ldots \ldots \ldots \ldots$

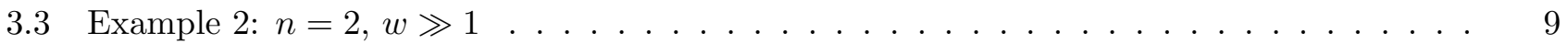

4 Evidence from the near horizon $\quad 11$

4.1 Superconformal quantum mechanics . . . . . . . . . . . . . . . . . . . . 12

4.2 Corrections to $A d S_{3}$ central charge $\ldots \ldots \ldots \ldots \ldots \ldots$

\begin{tabular}{|lr}
5 & Discussion \\
\hline
\end{tabular}

A Derivation of eq. (38) 15

\section{Introduction}

The success of string theory in providing microscopic understanding of the entropy of extremal black holes has been extended in recent times to extremal small black holes with at least two charges [1]-[7]. For supersymmetric two-charge black holes the statistical entropy coming from counting the bound state degeneracy has been checked to agree with the one calculated using Wald's entropy formula $[8]$ in higherderivative supergravity [5, 6]. Somewhat miraculously, the subleading terms have also been shown to agree in certain cases [3]. The entropy function formalism developed by Sen [6] to calculate Wald's entropy for extremal black holes has been investigated in other contexts in [10]-14].

The most studied extremal small black hole is the one made from a long heterotic string wrapping a circle carrying winding charge $w$ and momentum $n$, namely, the FP system. A simple scaling argument using Sen's entropy function formalism [5, 6], shows that incorporating string tree-level higher derivative corrections gives rise to an entropy proportional to $\sqrt{n w}$ in agreement with the microscopic state counting. A question that naturally arises is what happens if one includes higher 10-dimensional string-loop corrections as well in calculating Wald's entropy. Naively, one would not expect a simple answer to this question $^{1}$. We will show in this note that contrary to this naive intuition, a simple formula for the entropy emerges when the 10-dimensional string-loop corrections are included. The modified entropy formula predicted by the macroscopic scaling argument will be shown to behave like

$$
S_{B H} \sim \sqrt{a n w+b n},
$$

\footnotetext{
${ }^{1}$ In the context of supersymmetric black holes carrying three or more charges, this question was partly investigated in 9$]$
} 
for $n \gg w \gg 1$ with $a$ and $b$ being constants. There are three important points to note about this formula.

1. When the square-root is expanded, we get corrections proportional to powers of $\sqrt{n / w}$ which will be more dominant than the subleading terms arising from the Hardy-Ramanujan-Rademacher formula for the entropy of the long string which begin with $\log (n w)$. Hence, it is important to understand the terms arising from the 10-dimensional string-loop corrections.

2. The formula admits a non-zero formal limit when $w \rightarrow 0$ and we have a single charge. In this case, string-loop effects become very important.

3. Motivated by quantum entanglement arguments, Kallosh and Linde [15] proposed that the single charge black hole entropy could be interpreted as arising from non-normalizability of a qubit wavefunction and conjectured that the black hole entropy formula could be made more "universal" of the form $\sqrt{a q_{1} q_{2}+b q_{1}}$ for the two-charge case. Our findings nicely corroborate this conjecture.

Recently in Ref. [10] we have provided evidence for the existence of extremal small black holes with just one electric charge with D0-brane solution as the main example ${ }^{2}$. The microscopic and macroscopic entropies agreed only after including a certain subset of non-bound states in the counting. In general there are continuous families of classically supersymmetric states corresponding to separating the D0branes in the nine spatial directions in type IIA. In [10] we extracted those states from the continuous families which have $S O(9)$ invariance (that is, no separation among D0-branes) and associated them to the single centered D0-brane solution. This subset of all possible non-bound states was shown to give the correct behaviour of the leading entropy predicted by Wald's entropy formula. This is a rather surprising result as it has been believed that only the bound states contribute to the entropy of an extremal black hole. Thus it raises the question of whether one should include more than the bound state entropy for the extremal black holes with more than one charge too.

Motivated by the above discussion of the D0-brane we will include a subclass of the classically supersymmetric multi-string configurations to the set of microstates of the two-charge black hole considered above. We again set aside the zero modes corresponding to separating the centers of mass of the fundamental strings. By looking at two special examples in detail, we show that this gives results for the statistical entropy consistent with the scaling given by the entropy function formalism.

Since the entropy function formalism depends only on the near horizon geometry, it is expected that the statistical entropy should also emerge from microscopic state counting in a quantum theory living at the horizon, or equivalently on the boundary of the AdS geometry in the spirit of AdS/CFT correspondence (see for example [22]). We propose that the near horizon counting should respect the macroscopic scaling.

In this note we outline two computations to check this proposal. Firstly, we use the chiral primary partition function of superconformal quantum mechanics [20] of the D0-D4 $C Y_{3}$ black hole to demonstrate that its asymptotics exhibit similar scaling to our loop corrected macroscopic result for the entropy. Secondly, motivated by the work of Kraus and Larsen [16], we provide a central-charge argument which can lead to the same scaling as that we find using Sen's entropy function formalism.

\footnotetext{
${ }^{2}$ See also [11] for a discussion in AdS context.
} 
The rest of this note is organized as follows. In section 2, we use the entropy function formalism to provide a scaling argument for the string-loop corrected small black hole macroscopic entropy. In section 3, we provide a modification of the microscopic counting which gives rise to the same scaling. In section 4.1, we consider asymptotics of the partition function of chiral primaries in superconformal quantum mechanics and show that it is similar to our scaling prediction. In section 4.2, we consider loop corrections from the central charge viewpoint. We summarize in section 5 . In the appendix, we provide a derivation of a relation between the $A d S_{3}$ central charge and the black hole entropy used in section 4.2.

\section{A scaling argument}

In this section we will explore the consequences of including the, hitherto neglected, 10-dimensional stringloop corrections to the entropy of the two-charge FP black hole in heterotic string compactified on $T^{6}$. We use Sen's entropy function formalism [6] to calculate Wald's entropy of this black hole. The main steps in using this formalism are as follows:

- Assume a near horizon geometry of the form $A d S_{2} \times S^{d}$ and assume that the fields respect the isometries of the spacetime.

- Write down a function $f$ defined by $f=\int \Omega_{S^{d}} L$ where $L$ is the spacetime Lagrangian.

- Define electric charges $q_{i}$ by $q_{i}=\partial_{e_{i}} f$, where $e_{i}$ are the electric fields at the horizon. Take the Legendre transform of $f$ only with respect to $q_{i}$ 's (even in the presence of magnetic fields).

$$
F=2 \pi\left(q_{i} e_{i}-f\right)
$$

and extremize this function with respect to the radii of $A d S_{2}$ and $S^{d}$ and scalar fields. The value of $F$ at its extremum is the black hole entropy.

We will assume that the near horizon geometry is going to be $A d S_{2} \times S^{2}$ in the presence of higher derivative corrections in our context. The 10-dimensional action for heterotic string theory in string frame including the higher-loop corrections schematically takes the form

$$
\int d^{10} x \sqrt{-G}\left[e^{-2 \phi}(R+\cdots)+\sum_{n=0}^{\infty} e^{2 n \phi}[(n+1)-\text { loop terms }]\right]
$$

where $\cdots$ represent the $\alpha^{\prime}$ corrections at the tree-level. The string-loop terms start at the quartic order at 1-loop and we include all the $\alpha^{\prime}$ corrections at each order. This action can be dimensionally reduced on $T^{5} \times S^{1}$ with $T$ being the circle-size modulus and $T / S=g_{s}^{2}=e^{2 \phi}$ the 10-dimensional string coupling. This will give

$$
f\left(v_{1}, v_{2}, v_{3}, v_{4}, S, T\right)=S g\left(v_{1}, v_{2}, v_{3}=e_{1} T, v_{4}=e_{2} / T\right)+T h\left(v_{1}, v_{2}, v_{3}, v_{4}, \alpha=T / S\right)
$$


Here $v_{1}$ and $v_{2}$ denote the radii of $A d S_{2}$ and $S^{2}$ respectively while $e_{1}$ and $e_{2}$ denote the two electric fields of the Kaluza-Klein gauge fields coming from the 10-dimensional metric and the B-field respectively. Taylor expanding the function $h\left(v_{1}, v_{2}, v_{3}, v_{4}, \alpha\right)$ in powers of $\alpha$ accounts for all the higher string-loop corrections in $f$. To find the near horizon solution one has to extremize $f$ with respect to $S, T, v_{1}$ and $v_{2}$. The $S$ and $T$ equations of motion can be written as

$$
g-\alpha^{2} \partial_{\alpha} h=0, \quad\left(v_{3} \partial_{v_{3}}-v_{4} \partial_{v_{4}}+1\right)(g+\alpha h)=0
$$

The two electric charges, $q_{1}=T \partial_{v_{3}} f, q_{2}=\frac{1}{T} \partial_{v_{4}} f$ correspond to the momentum $n$ and winding $w$ of the fundamental string. These read

$$
q_{1}=S T \partial_{v_{3}} g+T^{2} \partial_{v_{3}} h, \quad q_{2}=\frac{S}{T} \partial_{v_{4}} g+\partial_{v_{4}} h
$$

which can be solved for $T$ and $\alpha$ to obtain

$$
T^{2}=\frac{q_{1} \partial_{v_{4}} g}{\left(q_{2}-\partial_{v_{4}} h\right) \partial_{v_{3}} g+\partial_{v_{3}} h \partial_{v_{4}} g}, \quad \alpha=\frac{\partial_{v_{4}} g}{q_{2}-\partial_{v_{4}} h} .
$$

Note that the right hand sides of these equations depend on $\alpha$ implicitly through $h$. The equations of motion for $v_{1}$ and $v_{2}$ are

$$
\partial_{v_{i}} g+\alpha \partial_{v_{i}} h=0
$$

for $i=1$ and 2 . Then the entropy function reads

$$
S_{B H}=2 \pi\left(e_{1} q_{1}+e_{2} q_{2}-f\right)=4 \pi \frac{v_{3} q_{1}}{T} .
$$

In obtaining the final expression we have used the equation of motion for $T$ and the expression for $q_{1}$. Now we can simply substitute the expression for $T$ from eq.(5D) in terms of the charges to arrive at

$$
S_{B H}=4 \pi \sqrt{q_{1}\left(a q_{2}+b\right)}
$$

where $a=v_{3}^{2} \partial_{v_{3}} g / \partial_{v_{4}} g$ and $b=a\left(\partial_{v_{3}} h \partial_{v_{4}} g-\partial_{v_{4}} h \partial_{v_{3}} g\right) / \partial_{v_{3}} g$. However, for the coefficients $a$ and $b$ to be universal we have to have $v_{i}, \partial_{v_{i}} g$ and $\partial_{v_{i}} h$ appearing in $a$ and $b$ to be charge independent. Since the eqs.(3) and (6) depend on $\alpha$ explicitly as well as implicitly through $h$ the coefficients in eq. (8) are not completely universal. Let us first consider two special cases where $a$ and $b$ do become universal.

- If we set $h=0$ then $b=0$ and the $\alpha$ dependence from eqs.(31) and (6) drops out. These equations allow us to solve for $v_{i}$ and hence $a$ becomes universal. In this case one recovers the result of Sen [5].

- If we set $q_{2}=0$ then $\alpha$ and therefore $h$ become charge independent. In this case too the eqs.(3) and (6) become charge independent. Then the unknown coefficient $b$ in the entropy becomes universal, thus recovering the result of [10]. 
Since we obtained eq.(2) from the effective action of the perturbative string, we have $\alpha \ll 1$. However since $\alpha$ can be determined through the second of eq. (41) it depends only on $q_{2}$. So the coefficients $a$ and $b$ in eq. (88) depend, at the most, on $q_{2}$ but not on $q_{1}$. Now we would like to argue that $a$ and $b$ are universal to the leading order in $\alpha$. For this it is sufficient to look at the entropy function including just the 1-loop terms. Then we have

$$
f\left(v_{1}, v_{2}, v_{3}, v_{4}\right)=\underbrace{S g\left(v_{1}, v_{2}, v_{3}, v_{4}\right)}_{\text {string tree-level }}+\underbrace{T h_{0}\left(v_{1}, v_{2}, v_{3}, v_{4}\right)}_{\text {string one-loop }}
$$

where $h_{0}=h(\alpha=0)$. This leads to the entropy

$$
S_{B H}=4 \pi \frac{q_{1} v_{3}}{T}=4 \pi \sqrt{q_{1} q_{2} v_{3} v_{4}-q_{1} v_{3} h_{0}}
$$

and the near horizon values of the scalar fields

$$
T=\sqrt{\frac{v_{3} q_{1}}{v_{4} q_{2}-h_{0}}}, \quad S=\frac{q_{2}-\partial_{v_{4}} h_{0}}{\partial_{v_{4}} g} \sqrt{\frac{v_{3} q_{1}}{v_{4} q_{2}-h_{0}}} .
$$

Now for $\alpha=T / S \ll 1$ or in other words when $q_{2} \gg 1$ we can treat the equations of motion obtained from (9) perturbatively in $\alpha$. This results in $\partial_{v_{1}} g=0, \partial_{v_{2}} g=0$ to the leading order. These together with $g=0$ and the perturbative approximation to the equation of motion for $T$ which is $v_{3} \partial_{v_{3}} g=v_{4} \partial_{v_{4}} g$ provide four equations independent of the charges to solve for $v_{1}, v_{2}, v_{3}$ and $v_{4}$. Furthermore, note that for the higher-derivative expansion to make sense we need $T \gg 1$ or in other words $q_{1} \gg q_{2}$. However, it is not necessary to impose this constraint to get the above scaling. Thus, we see that the scaling of the macroscopic entropy is indeed of the type given by equation eq.(10).

One would naively have expected, based on the tree-level result $S \sim \sqrt{q_{1} q_{2}}$, to go to zero in the $q_{2} \rightarrow 0$ limit and hence string coupling to blow up. However $S$ and $T$ remain sensible in the $q_{2} \rightarrow 0$ limit and scale the same way, i.e., $\sqrt{q_{1}}$. Thus the 10-dimensional string coupling is independent of the charges in this limit as $g_{s}^{2} \sim T / S \sim O(1)$. This suggests that in this limit, both $\alpha^{\prime}$ and $g_{s}$ corrections become equally important.

It is the opposite limit $q_{1} \rightarrow 0$ that appears problematic at first sight. This is not entirely unexpected as $T \rightarrow 0$ in this limit and hence the higher-derivative expansion breaks down. In general, when one dimensionally reduces an action, one misses terms in the lower dimensional theory which vanish on taking the decompactification limit. A well-known example is the dimensional reduction of the M-theory $R^{4}$ term which gives only the 1-loop type IIA $R^{4}$ term while the tree-level term arises due to Kaluza-Klein effects in the 11-dimensional theory on a circle [18. Such terms typically come with inverse powers of the compact volume so that on taking the decompactification limit, they vanish. In our context this must be the reason for the lack of T-duality invariance of the entropy. This may be cured by making the function $f$ invariant under T-duality $T \rightarrow 1 / T$ which we will turn to next. 
A T-dual invariant scaling: This motivates us to write a T-dual invariant entropy function of the form

$$
f=S g+\left(T+\frac{1}{T}\right) h_{0}
$$

keeping terms only upto the string 1-loop order. Repeating the above analysis for this function yields

$$
\begin{aligned}
S_{B H} & =4 \pi \sqrt{\left(q_{1} v_{3}-h_{0}\right)\left(q_{2} v_{4}-h_{0}\right)}, \\
T & =\sqrt{\frac{q_{1} v_{3}-h_{0}}{q_{2} v_{4}-h_{0}}}, \\
S & =\sqrt{\frac{\left(q_{1} q_{2} v_{4}-q_{1} h_{0}-\left(q_{1} v_{3}+q_{2} v_{4}-2 h_{0}\right) \partial_{v_{3}} h_{0}\right)\left(q_{1} \leftrightarrow q_{2}, v_{3} \leftrightarrow v_{4}\right)}{\partial_{v_{3}} g \partial_{v_{4}} g\left(q_{1} v_{3}-h_{0}\right)\left(q_{2} v_{4}-h_{0}\right)}} .
\end{aligned}
$$

This entropy is manifestly T-duality invariant, i.e., under $q_{1} \leftrightarrow q_{2}, v_{3} \leftrightarrow v_{4}$ as expected. In order to argue for (leading order) universality of $v_{i}$ there are now three possibilities: (a) $S \gg T \gg 1 / T$ so that $n \gg w \gg 1$ or $S \gg 1 / T \gg T$ so that $w \gg n \gg 1$, (b) $q_{1}=0$, (c) $q_{2}=0$. Note that when $q_{1}=q_{2}=0$ then, $h_{0}=0$ and $S_{B H}=0$ as it should be. We should however mention that the above T-duality invariant entropy function in eq.(12) is not derived from first principles and therefore should be considered as an illustrative toy model.

\section{Microstate re-counting}

We have seen that, when one includes string-loop corrections, the scaling of the entropy of the two-charge extremal black hole is different from the string tree-level one. One still needs to compute the universal numbers $a$ and $b$ that appear in eq.(8) with explicit higher derivative corrections. Unfortunately one does not have much control over the relevant higher derivative terms. Here we assume that $a$ and $b$ in eq. (8) are going to be non-zero. ${ }^{3}$ Such modification of the entropy cannot be accounted for just by counting the degeneracy of the bound states, namely, the Dabholkar-Harvey states in the first quantized string theory. This is so because the corrections to the leading entropy coming from the bound state counting depend only on the combination $q_{1} q_{2} \sim n w$ whereas string-loop corrections depend on $q_{1} / q_{2} \sim n / w$ as well.

Motivated by the discussion of the D0-charged black hole [10] in the introduction, we propose to include a subset of multi-strings states which carry exactly the same quantum numbers and are (at least) classically supersymmetric. In the case of the FP system there are continuous zero-modes corresponding to separating the centers of mass of different strings in a multi-string state spatially in the compact and non-compact directions. To do a counting of discrete states we suppress these modes and consider only one state in each such continuous family. Then the problem boils down to counting the number of ways one can distribute the total winding and momentum over several different (chiral and bosonic) strings.

\footnotetext{
${ }^{3}$ We provide evidence in favour of this assumption in section 5 .
} 


\subsection{Multi-string Partition function}

Let us write down a partition function including this discrete subset of the full set of multi-string states of the FP system. Recall that for the single string we have

$$
m(R)=\frac{n}{R}+\frac{R w}{\alpha^{\prime}}, \quad N:=N_{L}-1=n w
$$

where $N_{L}$ is the left-moving oscillator number. Then the single-string partition function is

$$
Z_{1}(q)=\prod_{k=1}^{\infty} \frac{1}{\left(1-q^{k}\right)^{24}}=\sum_{N=0}^{\infty} p_{24}(N) q^{N} .
$$

One can give a closed-form expression for the multi-string partition function that we seek as well. The result is as follows:

$$
Z(x, y)=f_{1}(x) f_{1}(y) f_{2}(x, y)
$$

where

$$
f_{1}(x)=\prod_{k=1}^{\infty} \frac{1}{1-x^{k}}=\sum_{k=0}^{\infty} p(n) x^{n}
$$

with $p(n)$ being the number of partitions of $n$ and

$$
f_{2}(x, y)=\prod_{k, l=1}^{\infty} \frac{1}{\left(1-x^{k} y^{l}\right)^{p_{24}(k l)}}
$$

with $p_{24}(n)$ being the number of 24-colored partitions of $n$, generated by

$$
\prod_{n=1}^{\infty} \frac{1}{\left(1-x^{n}\right)^{24}}=f_{24}(x)=\sum_{l=0}^{\infty} p_{24}(l) x^{l}
$$

To see this let us note that any multi-string state with total winding and momentum given by $n$ and $w$ has three types of constituent strings: (i) those carrying purely winding number, i.e, $n=0$, (ii) those carrying purely momentum number, i.e, $w=0$ and (iii) those with both winding and momentum non-zero. So the full partition function for multi-string states is given by the product of three pieces. The first two pieces are the multi-string partition functions of the first two species of strings listed above respectively. These are the same as the generating functions for number of partitions, namely, $f_{1}(x)$ and $f_{1}(y)$.

For the third piece, consider multi-string states with each constituent string having momentum $k$ and winding $l$. Since the component strings can be treated as indistinguishable bosons with each string having $p_{24}(k l)$ 'flavours', the degeneracy for a $p$-string state is given by the number of components of a symmetric rank- $p$ tensor whose indices can take $p_{24}(k l)$ values. This number is $\frac{\left(p_{24}(k l)-1+p\right) !}{p^{\prime}\left(p_{24}(k l)-1\right) !}$. Therefore the partition 
function for multi-string states with each string carrying charges $(k, l)$ is:

$$
\sum_{p=0}^{\infty} \frac{\left(p_{24}(k l)-1+p\right) !}{p !\left(p_{24}(k l)-1\right) !}\left(x^{k} y^{l}\right)^{p}=\left(1-x^{k} y^{l}\right)^{-p_{24}(k l)}
$$

Then the full partition function of the states with both non-zero charges is simply given by taking the product of terms in eq.(22) over non-zero $k$ and $l$ which gives $f_{2}(x, y)$. This completes the proof of the two-charge multi-string partition function of eq.(18).

We should point out that there is a BPS degeneracy of 16 for each 1/4-BPS state in the single string Hilbert space coming from the fermionic zero-modes in the right-moving sector of heterotic string and we have not taken these into account. If we are to include them as number of 'flavours' for each string then the full partition function will be $Z^{16}(x, y)$. It is not clear to us if one should include this degeneracy into the counting or not. However the results below can be modified easily to incorporate such an extra power and the qualitative features remain the same as without these extra states.

We should next analyze ${ }^{4}$ the partition function in eq.(18) to extract the statistical entropy. We will look at the special cases of $n=1,2$ and $w \gg 1$.

\subsection{Example 1: $n=1, w \gg 1$}

Consider the case when the total momentum $w \gg 1$ and winding $n=1$. The partition function of this case is given by the coefficient of $x$ in the full partition function which is

$$
f_{1}(y)\left[1+\sum_{k=1}^{\infty} p_{24}(k) y^{k}\right]=f_{1}(y) f_{24}(y)=\prod_{k=1}^{\infty} \frac{1}{\left(1-y^{k}\right)^{25}}=\sum_{w=0}^{\infty} p_{25}(w) y^{w}
$$

So the degeneracy is simply given by $p_{25}(w)$ which in the large $w$ limit can be easily shown to yield

$$
\exp \left(\pi \sqrt{w} \sqrt{\underbrace{16}_{\alpha^{\prime}}+\underbrace{2 / 3}_{\text {string loop }}}\right) .
$$

This gives entropy $\pi \sqrt{w} \sqrt{16+2 / 3}$ consistent with that in eq.(8).

\subsection{Example 2: $n=2, w \gg 1$}

Now consider the case $n=2$ and $w \gg 1$. The full partition function can be expanded to keep all terms of order $x^{2}$ :

$$
\prod_{k=1}^{\infty} \frac{1}{1-y^{k}}\left[2+\sum_{m=1}^{\infty} p_{24}(m) y^{m}+\frac{1}{2} \sum_{m=1}^{\infty}\left[p_{24}(m)+p_{24}^{2}(m)\right] y^{2 m}\right.
$$

\footnotetext{
${ }^{4}$ We thank J. Lucietti for discussions in section 3.2 and 3.3 .
} 


$$
\left.+\sum_{\substack{m<n \\ m, n=1}}^{\infty} p_{24}(m) p_{24}(n) y^{m+n}+\sum_{n=1}^{\infty} p_{24}(2 n) y^{n}\right] x^{2}
$$

Noticing that one can identify most of the terms here as those in the expansion of

$$
\frac{1}{2}\left(1+\sum_{m=1}^{\infty} p_{24}(m) y^{m}\right)\left(1+\sum_{n=1}^{\infty} p_{24}(n) y^{n}\right)=\frac{1}{2}+\sum_{m=1}^{\infty} p_{24}(m) y^{m}+\frac{1}{2} \sum_{m, n=1}^{\infty} p_{24}(m) p_{24}(n) y^{m+n}
$$

and then recognizing this as simply $\frac{1}{2}\left(\prod_{k=1}^{\infty} \frac{1}{\left(1-y^{k}\right)^{24}}\right)^{2}=\frac{1}{2}\left(\prod_{k=1}^{\infty} \frac{1}{1-y^{k}}\right)^{48}$ we can rewrite the above $n=2$ partition function as

$$
\prod_{l=1}^{\infty} \frac{1}{1-y^{l}}\left[\frac{1}{2}\left(\prod_{k=1}^{\infty} \frac{1}{1-y^{k}}\right)^{48}+\frac{1}{2}\left(1+\sum_{m=1}^{\infty} p_{24}(m) y^{2 m}\right)+\left(1+\sum_{n=1}^{\infty} p_{24}(2 n) y^{n}\right)\right] x^{2}
$$

Then the coefficient of $y^{w}$ reads:

$$
\frac{1}{2} p_{49}(w)+\frac{1}{2} \sum_{k=0}^{w} \delta_{\left[\frac{w-k}{2}\right]-\frac{w-k}{2}} p(k) p_{24}\left(\frac{w-k}{2}\right)+\sum_{k=0}^{w} p(k) p_{24}(2 w-2 k) .
$$

The last term is the one coming from multi-string states with both units of momentum carried by one single string. The 2 nd term goes at most like $p(w)$ or $p_{24}(w / 2)$ and so can be dropped in comparison to the first and the third. We will now see that the 1st and 3rd term behave the same way for large $w$. The proof is as follows. First note that

$$
g(x)=\frac{1}{2}\left(f_{24}(\sqrt{x})+f_{24}(-\sqrt{x})\right)=\sum p_{24}(2 n) x^{n} .
$$

Then it is easy to see that the 3rd term simply becomes the coefficient of $x^{w}$ in $f(x) g(x)$. Let us find out how $f(x) g(x)$ behaves near $x \rightarrow 1$ which is where we expect the maximum contribution. $f(x) g(x)$ is made of two terms. The first term can be written as [19]

$$
\begin{aligned}
\exp \left(-\sum_{n=1}^{\infty} \log \left(1-x^{n}\right)-24 \sum_{n=1}^{\infty} \log \left(1-x^{n / 2}\right)\right) & =\exp \left(\sum_{m, n=1}^{\infty} \frac{x^{m n}}{m}+24 \sum_{m, n=1}^{\infty} \frac{x^{m n / 2}}{m}\right) \\
=\exp \left(\sum_{m}\left[\frac{x^{m}}{m\left(1-x^{m}\right)}+24 \frac{x^{m / 2}}{m\left(1-x^{m / 2}\right)}\right]\right) & \sim \exp \left(\frac{1}{1-x} \sum_{m} \frac{1}{m^{2}}+\frac{24 \times 2}{m^{2}}\right) \\
& =\exp \left(\frac{1}{1-x} \frac{49 \pi^{2}}{6}\right) .
\end{aligned}
$$

The second term is

$$
\exp \left(-\sum_{n=1}^{\infty} \log \left(1-x^{n}\right)-24 \sum_{n=1}^{\infty} \log \left(1-(-1)^{n} x^{n / 2}\right)\right)
$$


and following the same steps as above we get

$$
\exp \left(\sum \frac{x^{m}}{m\left(1-x^{m}\right)}+24 \frac{(-1)^{m} x^{m / 2}}{m\left(1-(-1)^{m} x^{m / 2}\right)}\right) .
$$

Now writing $(-1)^{m}=e^{\pi i m}$ and putting $e^{2 \pi i} x=1+h$ we can show that this term behaves exactly like the first term in $g(x) f(x)$. These manipulations are similar to those found in [19] and it would be nice to have a proof based on modular properties of the functions also. We will not attempt this here.

Now it is easy to see that the third term will behave in the same manner for large $w$ as the first term. Hence we conclude that for the $n=2, w \gg 1$ case (similarly for $w=2, n \gg 1$ with $w, n$ interchanged), the entropy is given by

$$
\pi \sqrt{w} \sqrt{16 \times 2+2 / 3} .
$$

We are investigating the generalization of this formula to all $n$ separately [24]. The result to be expected for large $n, w$ may be

$$
S_{\text {stat }}=\pi \sqrt{16 n w+\frac{2}{3} n+\frac{2}{3} w},
$$

or some other formula which reduces to the cases studied above. It will be interesting to see exactly which T-duality invariant formulae is correct by considering the case of general $n$ and $w$ on the counting side.

\section{Evidence from the near horizon}

Since Wald's entropy for an extremal black hole depends on the lagrangian evaluated on the near horizon geometry alone, it is natural to suspect that it can be evaluated by a counting of states in string theory on the near horizon geometry of the black hole (see for example [22]). However we have argued so far that the entropy of the two-charge black holes could include contributions from a subset of non-bound states as well.

If true, these proposals should mean that the full entropy of an extremal black hole coming from counting the corresponding BPS states in the holographically dual superconformal quantum theory on the boundary of the near horizon geometry should also include the contributions coming from the subset of non-bound states like those considered in section 3. We suggest that this is indeed the case. In what follows we sketch two computations towards providing evidence for our modified scaling of the entropy of eq.(8). For this, note that, even though the scaling eq.(8) is derived in heterotic on $T^{6}$ it should be valid for black holes in all of the following three duality frames.

1. F-strings with momentum and winding in heterotic on $T^{6}$,

2. D0-D4 brane system in type IIA on $K 3 \times T^{2}$,

3. D1-D5 brane system in type IIB on $K 3 \times T^{2}$.

We ask whether the entropy scaling $\sqrt{n(a w+b)}$ could be seen by counting the near horizon microstates in any/all of these duality frames. Good laboratories to answer this question are the holographic duals 
of string theories in the respective near horizon geometries. In particular, the superconformal quantum mechanics of 22 related to the second duality frame and the 2-dimensional D1-D5 CFT relevant for the third duality frame. Below we sketch two computations towards extracting information of microstates which cannot be associated with the bound states (as seen from the asymptotic flat space) from these boundary theories.

\subsection{Superconformal quantum mechanics}

First we consider the superconformal quantum mechanics describing $D 0$ branes in the $A d S_{2} \times S^{2} \times C Y_{3}$ attractor geometry of a black hole in type IIA with $D 4$-branes on the $C Y_{3}$. This quantum mechanics has a class of chiral primaries which have been identified with the microstates of the black hole. It was shown in [22] that the Bekenstein-Hawking area law for a large black hole could be reproduced exactly from the asymptotic degeneracy of these chiral primaries. The chiral primary generating function was argued to be

$$
Z=\prod_{n=1}^{\infty} \frac{\left(1+q^{n}\right)^{h_{1}+h_{3}}}{\left(1-q^{n}\right)^{h_{0}+h_{2}}}=\sum_{N=0}^{\infty} q^{N} d(N),
$$

where

$$
\begin{aligned}
& h_{0}=D+\frac{1}{12} c_{2} \cdot P, \quad h_{1}=3 D-\frac{3}{4} c_{2} \cdot P-\frac{\chi}{2} \\
& h_{2}=3 D-\frac{3}{4} c_{2} \cdot P+\frac{\chi}{2}, \quad h_{3}=D+\frac{1}{12} c_{2} \cdot P,
\end{aligned}
$$

with $D=D_{A B C} P^{A} P^{B} P^{C}$. The $D_{A B C}$ are the intersection numbers and $P^{A}$ are the D4-brane charges. The above partition function is the same as that for a CFT with $h_{1}+h_{3}$ fermions and $h_{0}+h_{2}$ bosons. The asymptotic value of $d(N)$ in equation (34) can be shown to be

$$
d(N) \sim \exp \left(2 \pi \sqrt{N\left(D-\frac{1}{6} c_{2} \cdot P+\frac{\chi}{24}\right)}\right),
$$

In [22], the authors only considered the leading term proportional to $D$. However, in the two-charge small black hole example we have to set $D=0$. Then we arrive at the relevant formula for the statistical entropy which is clearly of the form $\sqrt{n(w+b)}$. Furthermore, note that if we replace $C Y_{3}$ by $K 3 \times T^{2}$ we have $c_{2} \cdot P=-24 w$ with $\chi=24$. This results in

$$
S_{B H}=\pi \sqrt{16 n w+4 n} .
$$

Curiously, this naive replacement produces the correct leading order term $4 \pi \sqrt{n w}$ for the two-charge black hole in type IIA on $K 3 \times T^{2}$ with D0 and D4-branes. The entropy of this system was worked out in a different way in 23] although the authors obtained only the leading term in their approach.

It would be desirable to repeat the calculation for $K 3 \times T^{2}$ rigorously. If for example, one could argue convincingly that the counting should be such that $b=0$ one would have the basis of a non-renormalization 
theorem suggesting no loop corrections to the entropy formula which would definitely merit attention. ${ }^{5}$

We note however, that IIA on $C Y_{3}$ is related by duality to heterotic on $K 3 \times T^{2}$ and hence we have a prediction in eq. (37) for the microscopic side for this theory. The prepotential for heterotic on $K 3 \times T^{2}$ was given, for example, in [9] and it would be very interesting to see if the macroscopic calculation can be done in this case.

In the next subsection we pose the same question in terms of the central charge of the CFT living on the boundary of $A d S_{3} \times S^{2}$ which can be obtained by lifting the near horizon geometry of the two-charge black in the third duality frame (type IIB on $K 3 \times T^{2}$ with D1 and D5-branes) to five dimensions.

\subsection{Corrections to $A d S_{3}$ central charge}

To begin with one has to establish a connection between the central charge of $A d S_{3}$ and the entropy of two-charge small black holes. We will start with the D1-D5-P system and assume that the relation derived between the central charge and the black hole entropy continues to hold even when one of the three charges is switched off and we have a small black hole.

Since the central charge of $A d S_{3}$ is inversely proportional to $G_{3}$, the 3 -dimensional Newton constant and the entropy of the black hole is inversely proportional to the 2-dimensional Newton constant $G_{2}=G_{3} / T$, we expect the central charge $c$ of $A d S_{3}$ to be proportional to $F / T$, where $F$ is the entropy function at its extremum, i.e., the black hole entropy. It can be shown that this is indeed the case. We get

$$
F=\frac{\pi}{6} c(l) \tilde{T}
$$

where $T=l^{2} \tilde{T}$ and $F$ and $c$ are evaluated at their extremum. Please see the appendix for a derivation of eq.(38).

This relation is expected to hold for large $T$ as it involves dimensional reduction. Chern-Simons terms can be absorbed into the definition of $c(l)$. Although the formula (38) was derived in the appendix with the 3-charge black hole in mind we expect it to hold for the two-charge small black hole as well. Let us cross-check this formula (38) with a known result for the two-charge small black hole. We know that for the black hole in heterotic on $T^{6}, F=4 \pi \sqrt{n w}, \tilde{T}=\sqrt{n / w}$. Hence we expect from (38) that $c=24 w$. This is precisely the central charge ${ }^{7}$ obtained from holographic Weyl anomaly related arguments by Kraus and Larsen $[16]$.

We now expect that if there are string-loop corrections to the black hole entropy, these can be mapped (at least when $T$ is large) to the corrections to the central charge in $A d S_{3}$ associated with the black hole.

\footnotetext{
${ }^{5}$ Noting that the Betti numbers for $K 3, b_{0}=1, b_{1}=0, b_{2}=22, b_{3}=0, b_{4}=1$ and taking into account the Landau degeneracy due to the magnetic field into account, our preliminary analysis ${ }^{6}$ suggests assigning an entropy of $4 \pi \sqrt{n(w+1 / 2)}$ to the $K 3 \times T^{2}$ small black hole. Similarly if we take $C Y 3$ to be $T^{6}$ we get $2 \sqrt{2} \pi \sqrt{n(w+1)}$. Curiously, both these formulae can be written as $\pi \sqrt{n\left(c_{L} w+c_{R}\right)}$. From our multi-string counting this requires replacing $f_{1}$ by $f_{1}^{c_{R}}$. We hope to return to these issues in a future work.

${ }^{7}$ More precisely, in presence of Chern-Simons terms the central charge splits into $c_{L}$ and $c_{R}$ with $c_{L} \neq c_{R}$. In the black hole entropy considered in the literature, only $c_{L}$ or $c_{R}$ enters. In the notation of [16], this is the left-moving central charge.
} 
Let us give a heuristic explanation how such corrections could arise macroscopically. It was shown in [16, 17] that the central charge in higher derivative gravity is given by

$$
\begin{aligned}
c & =\frac{l_{A d S}}{2 G_{3}} g_{\mu \nu} \frac{\partial L_{3}}{\partial R_{\mu \nu}} \\
& \sim \frac{l_{A d S}}{l_{P}}\left(c_{0}+g_{s}^{2} c_{1}+g_{s}^{4} c_{2}+\cdots\right),
\end{aligned}
$$

where $l_{P} \propto g_{s}^{2}$ is the Planck length and we anticipate higher-derivative corrections in the 3-dimensional Lagrangian to be weighted by $l_{P} / l_{A d S}$. Now noting that with $l_{\text {string }}=1$ we expect for small black holes $l_{A d S} \sim O(1)$ and using Sen's scaling result to put $g_{s}^{2}=1 / w$ we find

$$
c \sim c_{0} w+c_{1}+c_{2} / w+\cdots
$$

and hence the black hole entropy can sum to behave like

$$
S_{B H} \sim \sqrt{n(a w+b)}
$$

as our scaling arguments predict. It will be interesting to explicitly compute the corrections to the central charge using the technology developed in [21] and verify that it indeed respects the above scaling.

\section{Discussion}

We have shown using general scaling arguments and incorporating the 10-dimensional string-loop higher derivative corrections that the entropy of the two-charge extremal small black holes in heterotic string on $T^{6}$ can get corrections that cannot be accounted for by the standard bound state counting alone. We found that the modified entropy behaves as

$$
S_{B H} \sim \sqrt{q_{1}\left(a q_{2}+b\right)}
$$

where $a$ and $b$ become independent of charges in specific limits, namely $q_{1} \gg q_{2} \gg 1$ or if one of the charges is set to zero. On the microscopic counting side, we included by hand a certain subset of multistring configurations and found for two separate cases that their counting also has the same behaviour as predicted by the scaling argument. We presented a conjecture that for a two-charge small black hole carrying momentum $n$, winding $w$ and $n \gg w \gg 1$, the entropy should be given by

$$
S_{\text {stat }}=\pi \sqrt{16 n w+\frac{2}{3} w}
$$

We further argued that Wald's entropy including string-loop corrections can be evaluated by counting appropriate BPS states in the near horizon geometry of the black hole. We have provided partial evidence for this by considering the chiral primary states in the holographically dual superconformal quantum 
mechanics of [22] on the boundary of the near horizon geometry of CY black holes in type IIA. We also sketched a computation of string loop corrections to the central charge of the dual CFT of $A d S_{3}$ and its relation to the entropy of the two-charge black holes. It will be interesting to see if one can recover the full partition function that we wrote down from a counting completely within the near horizon theory or its holographic dual. If, on the other hand, one could convincingly demonstrate that the microstate counting dictates $b=0$ in any of the duality frames, then it would mean that macroscopic string loop corrections would be absent, which would merit further attention.

Finally, it will be interesting to see if there are potential corrections to the entropy of some of the large extremal black holes as well in string theory which may come from microstates other than the standard bound states in analogy to the multi-string states considered in section 3. For instance, it is possible that Wald's entropy of the D1-D5-P black hole admits corrections so that when one of the charges is set to zero one recovers the entropy of the two-charge black hole that we considered here. We hope to return to some of these questions in the near future.

\section{A Derivation of eq. $(38)$}

Here we will follow [20] closely. The starting point is the $A d S_{3}$ part of the near horizon geometry of the D1-D5-P system which is given by

$$
d s_{3}^{2}=l^{2} T^{2}\left(d x_{5}+\frac{d t}{R}\right)^{2}+\frac{U^{2}}{l^{2}}\left(R^{2} d x_{5}^{2}-d t^{2}\right)+l^{2} \frac{d U^{2}}{U^{2}},
$$

where the asymptotic radius of $S^{1}$ around which momentum $n$ flows is given by $R$ and the variables $T, l$ are defined as

$$
T=\sqrt{\frac{n}{k}}, \quad l^{4}=g_{6}^{2} k, \quad k=Q_{1} Q_{5}
$$

where $g_{6}$ is the six-dimensional coupling and the quantity $T$ is the near horizon value of the radius of $S^{1}$. To get $A d S_{2}$ from $A d S_{3}$ we write the metric as

$$
d s_{3}^{2}=d s_{2}^{2}+l^{2} e^{2 \psi}\left(d x_{5}+A_{t} d t\right)^{2}
$$

where after rescaling $t$ by $R, U$ by $l^{2} / R$ and performing the change of variables $U^{2}=4 r$

$$
\begin{aligned}
e^{2 \psi} & =T^{2}+4 r \\
A_{t} & =T^{2} e^{-2 \psi} \\
d s_{2}^{2} & =-\frac{16}{l^{2}\left(T^{2}+4 r\right)} r^{2} d t^{2}+l^{2} \frac{d r^{2}}{r^{2}}+l^{2} e^{2 \psi}\left(d x_{5}+A_{t} d t\right)^{2} .
\end{aligned}
$$


The $A d S_{2}$ factor is obtained by taking a "near near-horizon" limit $U / T \rightarrow 0$. This yields after rescaling $t$ by $4 /\left(l^{2} T\right)$

$$
d s_{3}^{2}=l^{2}\left(-r^{2} d t^{2}+\frac{d r^{2}}{r^{2}}\right)+l^{2} e^{2 \psi}\left(d x_{5}+A_{t} d t\right)^{2}
$$

where now

$$
A_{t}=\frac{l^{2}}{4} \frac{T^{3}}{T^{2}+4 r} \approx \frac{l^{2}}{4} T\left(1-\frac{4 r}{T^{2}}\right)
$$

giving $F_{r t}=-l^{2} / T=e$. Following Kraus and Larsen [16], Sahoo and Sen [13], we define the "centralcharge" function $c$ as follows. From equation (46)

$$
\sqrt{-\operatorname{det} G}=l^{3} T
$$

then defining

$$
f_{0}=2 \pi \sqrt{-\operatorname{det} G} L_{0}^{(3)}=-\frac{c(l)}{24} \frac{T}{l^{2}},
$$

we get following Sen's entropy function analysis, the entropy function

$$
F=2 \pi\left(q e-f_{0}\right)=2 \pi\left(\frac{q}{\tilde{T}}+\frac{c(l)}{24} \tilde{T}\right)
$$

where $T=l^{2} \tilde{T}$ and extremising with respect to $\tilde{T}, l$ yields

$$
F=\frac{\pi}{6} c(l) \tilde{T}
$$

all evaluated at the extremum. This gives us our proposed relation $c \sim F / T$ with proportionality constant $6 / \pi$.

\section{Acknowledgements}

We thank Allen Chen, Daniel Cremades, Atish Dabholkar, Michael Green, James Lucietti, Gautam Mandal, Samir Mathur, Rob Myers, Annamaria Sinkovics, Julian Sonner, Ashoke Sen and David Tong for discussions. AS acknowledges support from PPARC and Gonville and Caius college, Cambridge. NVS would like to thank DAMTP and the Indian research Institutes IoPB, IISc, IITM, IMSc, HRI, IACS and TIFR for hospitality at various stages of this work.

\section{References}

[1] A. Dabholkar, "Exact counting of black hole microstates," Phys. Rev. Lett. 94, 241301 (2005) arXiv:hep-th/0409148.

[2] G. L. Cardoso, B. de Wit, J. Kappeli and T. Mohaupt, "Supersymmetric black hole solutions with $R^{2}$ interactions," arXiv:hep-th/0003157. A. Dabholkar, R. Kallosh and A. Maloney, "A stringy cloak 
for a classical singularity," JHEP 0412, 059 (2004) arXiv:hep-th/0410076. V. Hubeny, A. Maloney and M. Rangamani, "String-corrected black holes," JHEP 0505, 035 (2005) arXiv:hep-th/0411272. B. de Wit, "Introduction to black hole entropy and supersymmetry," arXiv:hep-th/0503211. B. de Wit, "Supersymmetric black holes," arXiv:hep-th/0511261. T. Mohaupt, "Strings, higher curvature corrections, and black holes," arXiv:hep-th/0512048. G. L. Cardoso, B. de Wit, J. Kappeli and T. Mohaupt, "Black hole partition functions and duality," arXiv:hep-th/0601108.

[3] A. Dabholkar, F. Denef, G. W. Moore and B. Pioline, "Precision counting of small black holes," JHEP 0510, 096 (2005) arXiv:hep-th/0507014.

[4] A. Sen, "Extremal black holes and elementary string states," Mod. Phys. Lett. A 10, 2081 (1995) arXiv:hep-th/9504147.

[5] A. Sen, "Black holes, attractors, elementary strings etc.," Lectures given at the Perimeter Institute, Canada (2005).

[6] A. Sen, "Stretching the horizon of a higher dimensional small black hole," JHEP 0507, 073 (2005) arXiv:hep-th/0505122. A. Sen, "Black hole entropy function and the attractor mechanism in higher derivative gravity," arXiv:hep-th/0506177. A. Sen, "Entropy function for heterotic black holes," arXiv:hep-th/0508042,

[7] A. Sen, "How does a fundamental string stretch its horizon?," JHEP 0505, 059 (2005) arXiv:hep-th/0411255. A. Sen, "Black holes and the spectrum of half-BPS states in N $=4$ supersymmetric string theory," arXiv:hep-th/0504005.

[8] R. M. Wald, "Black hole entropy in the Noether charge," Phys. Rev. D 48, 3427 (1993) arXiv:gr-qc/9307038. T. Jacobson and R. C. Myers, "Black hole entropy and higher curvature interactions," Phys. Rev. Lett. 70, 3684 (1993) arXiv:hep-th/9305016. T. Jacobson, G. Kang and R. C. Myers, "Black hole entropy in higher curvature gravity," arXiv:gr-qc/9502009.

[9] K. Behrndt, G. Lopes Cardoso, B. de Wit, R. Kallosh, D. Lust and T. Mohaupt, "Classical and quantum N = 2 supersymmetric black holes," Nucl. Phys. B 488, 236 (1997) arXiv:hep-th/9610105.

[10] A. Sinha and N. V. Suryanarayana, "Extremal single-charge small black holes: Entropy function analysis," Class. Quant. Grav. 23, 3305 (2006) arXiv:hep-th/0601183.

[11] N. V. Suryanarayana, "Half-BPS giants, free fermions and microstates of superstars," JHEP 0601, 082 (2006) arXiv:hep-th/0411145.

[12] A. Ghodsi, " $R^{4}$ corrections to D1-D5-P black hole entropy from entropy function formalism," arXiv:hep-th/0604106.

[13] B. Sahoo and A. Sen, "Higher derivative corrections to non-supersymmetric extremal black holes in $\mathrm{N}=2$ supergravity," arXiv:hep-th/0603149.

[14] R. G. Cai and D. W. Pang, "Entropy Function for 4-Charge Extremal Black Holes in Type IIA Superstring Theory," arXiv:hep-th/0606098. B. Chandrasekhar, "Born-Infeld corrections to the en- 
tropy function of heterotic black holes," arXiv:hep-th/0604028, S. Parvizi and A. Tavanfar, "Partition function of non-supersymmetric black holes in the supergravity limit," arXiv:hep-th/0602292 B. Chandrasekhar, S. Parvizi, A. Tavanfar and H. Yavartanoo, "Non-supersymmetric attractors in $R^{2}$ gravities," arXiv:hep-th/0602022, M. Alishahiha and H. Ebrahim, "New Attractor, Entropy Function and Black Hole Partition Function," arXiv:hep-th/0605279. P. Prester, "Lovelock type gravity and small black holes in heterotic string theory," JHEP 0602, 039 (2006) arXiv:hep-th/0511306.

[15] R. Kallosh and A. Linde, "Strings, black holes, and quantum information," arXiv:hep-th/0602061

[16] P. Kraus and F. Larsen, "Microscopic black hole entropy in theories with higher derivatives," JHEP 0509, 034 (2005) arXiv:hep-th/0506176. P. Kraus and F. Larsen, "Holographic gravitational anomalies," JHEP 0601, 022 (2006) arXiv:hep-th/0508218.

[17] H. Saida and J. Soda, "Statistical entropy of BTZ black hole in higher curvature gravity," Phys. Lett. B 471, 358 (2000) arXiv:gr-qc/9909061.

[18] M. B. Green, M. Gutperle and P. Vanhove, "One loop in eleven dimensions," Phys. Lett. B 409, 177 (1997) arXiv:hep-th/9706175.

[19] M. B. Green, J. H. Schwarz and E. Witten, "Superstring Theory. Vol. 1: Introduction," Cambridge, Uk: Univ. Pr. (1987).

[20] A. Strominger, "AdS $S_{2}$ quantum gravity and string theory," JHEP 9901, 007 (1999) arXiv:hep-th/9809027.

[21] A. Giveon, D. Kutasov and N. Seiberg, "Comments on string theory on AdS(3)," Adv. Theor. Math. Phys. 2, 733 (1998) arXiv:hep-th/9806194. D. Kutasov and N. Seiberg, "More comments on string theory on $A d S_{3}$," JHEP 9904, 008 (1999) arXiv:hep-th/9903219. A. Giveon and D. Kutasov, "Notes on $A d S_{3}, "$ Nucl. Phys. B 621, 303 (2002) arXiv:hep-th/0106004.

[22] D. Gaiotto, A. Strominger and X. Yin, "Superconformal black hole quantum mechanics," JHEP 0511, 017 (2005) arXiv:hep-th/0412322.

[23] S. Kim and J. Raeymaekers, "Superconformal quantum mechanics of small black holes," JHEP 0508, 082 (2005) arXiv:hep-th/0505176.

[24] J. Lucietti, A. Sinha and N. V. Suryanarayana, work in progress. 